\title{
Completely Thoracoscopic Diaphragmatic Plication
}

\author{
Gregor J. Kocher ${ }^{1} \cdot$ Adrian Zehnder $^{1} \cdot$ Ralph A. Schmid $^{1}$
}

(C) Société Internationale de Chirurgie 2016

\begin{abstract}
Background Thoracoscopic diaphragmatic plication for diaphragmatic paralysis with consecutive eventration and respiratory compromise is a desirable alternative to standard thoracotomy. Since minimally invasive techniques usually involve suturing of the diaphragm, most surgeons use a video-assisted approach with a minithoracotomy. Herein we describe our completely thoracoscopic technique for diaphragmatic plication including outcome.

Methods We present our technique and experience for completely thoracoscopic diaphragmatic plication for the treatment of symptomatic diaphragmatic paralysis in six consecutive patients. The surgical technique basically consisted of stapling of the abundant diaphragm and reinforcement of the staple line using a self-locking thread. Primary outcome measure was the postoperative result (flattened diaphragm) and resolution of symptoms. Secondary outcome was improvement of lung function values 3 months after surgery.

Results Between June 2015 and March 2016, six patients have been operated for symptomatic diaphragmatic paralysis, with one of them suffering from additional transdiaphragmatic hernia. Flattening of the diaphragm was achieved in all 6 patients with resolution of their pre-existing symptoms within days after surgery and without any surgical complications. Lung function volumes measured 3 months postoperative improved markedly with an increase in FEV1 as well as FVC of $540 \mathrm{ml}(\mathrm{SD} \pm 193 \mathrm{ml})$ and $776 \mathrm{ml}(\mathrm{SD} \pm 121 \mathrm{ml})$, respectively.

Conclusions In our experience, the presented technique is a safe and simple minimally invasive way to perform a completely thoracoscopic diaphragmatic plication with excellent results so far.
\end{abstract}

\section{Introduction}

Diaphragmatic plication in adults is mostly performed for eventration with consecutive shortness of breath in patients suffering from phrenic nerve paralysis. The most common

Electronic supplementary material The online version of this article (doi:10.1007/s00268-016-3789-2) contains supplementary material, which is available to authorized users.

\section{Gregor J. Kocher}

gregor.kocher@insel.ch

1 Division of General Thoracic Surgery, Bern University Hospital, University of Bern, Bern, Switzerland cause for unilateral phrenic nerve paralysis is traumatic (including postsurgical), but also idiopathic palsy is not an uncommon finding [1]. The diaphragm can be surgically plicated in order to allow lung re-expansion on the affected side, which is generally performed through a low lateral thoracotomy. Several authors have described different techniques in order to allow a minimally invasive approach, but since suturing and suture tension are important cornerstones of the procedure, most surgeons still prefer a video-assisted approach involving a minithoracotomy [2].

Although the technique has been refined to a completely thoracoscopic approach by others [3], there are still technical difficulties that prevented a wide acceptance of such 
completely thoracoscopic approaches. Also the use of knifeless endostapling devices has been described before [4], but most surgeons do not use the stapling devices since they are concerned whether stapled suture lines of the diaphragm could withstand the intraabdominal pressure.

Herein we describe a simple and safe technique for a completely thoracoscopic diaphragmatic plication using an endostapler and an additional running suture to secure the staple line.

\section{Materials and methods}

Between June 2015 and March 2016, six patients have been operated for symptomatic diaphragmatic paralysis at our thoracic surgical unit. Informed consent was obtained from all patients before study inclusion. All patients presented with dyspnea on exertion $(n=5)$ or even the need for continuous oxygen treatment $(n=1)$ and showed a unilaterally elevated hemidiaphragm on routine chest X-ray. Diaphragmatic palsy was confirmed by a sniff-test under fluoroscopic control. Median patient age was 52 (range 48-86 years) with diaphragmatic paralysis being present on either left $(n=3)$ or right side $(n=3)$. Five patients were male, suffering from posttraumatic $(n=3)$, postoperative $(n=1)$ or idiopathic $(n=1)$ phrenic nerve paralysis, respectively. One patient was an 86-year-old lady suffering from idiopathic phrenic nerve paralysis with diaphragmatic eventration, Chilaiditi's syndrome and additional transdiaphragmatic intercostal hernia with colonic contents on the right side. Due to dyspnea and desaturation even at rest, she was dependent on a continuous oxygen treatment (1-2 L nasal per minute) throughout day and night.

\section{Surgical technique}

For the operation, the patient is intubated with a left-sided double-lumen tube and put in a lateral decubitus position, and furthermore, the patient is tilted in an approximately $30^{\circ}$ anti-Trendelenburg position. First a 5-mm air-sealed port is placed in the 6th intercostal space in the midaxillary line. After reassuring the correct position of the trocar in the pleural space by introducing a rigid 5-mm 30-angled thoracoscope, $\mathrm{CO} 2$ insufflation is started aiming at an intrathoracic pressure of $8 \mathrm{~mm}$ of mercury in order to further push down the diaphragm. Under thoracoscopic vision, another $5 \mathrm{~mm}$ port is placed in the 9th intercostal space in the midaxillary line and another $12 \mathrm{~mm}$ access port is introduced further posterior below the costal arch (Fig. 1). The diaphragm is then rolled up on a grasper until the desired tightness of the diaphragm is reached. The abundant diaphragm is stapled stepwise from postero-laterally to anteromedially with an endostapler (Endo $\mathrm{Gia}^{\mathrm{TM}}$, purple, $60 \mathrm{~mm}$ with Tri-Staple ${ }^{\mathrm{TM}}$ Technology, Covidien Switzerland LTD) (Video 1). After having removed the resected part of the diaphragm (Fig. 1), the staple line is reinforced with a running suture. In order to simplify the procedure, a self-locking barbed thread (V-loc 1, non-absorbable (polybutester)) was used for reinforcement of the staple line (Video 2). At the end of the procedure, a chest drain is inserted (20 Fr). The chest drain is usually removed on the first postoperative day.

\section{Results}

Mean operative time for diaphragmatic plication only ( $n=5$ ) was $67 \mathrm{~min}$ (range 50-90 min), and adequate flattening of the diaphragm with improved expansion of the
Fig. 1 Patient in right lateral decubitus position with the spine on the right side of the picture. One $5 \mathrm{~mm}$ port in the 6 th intercostal space in the anterior axillary line and one $5 \mathrm{~mm}$ port in the 9 th intercostal space in the posterior axillary line. The 12-mm port (with chest tube) was placed just below the tenth rib in the midaxillary line (a). The interrupted lines mark the location of the scapula and the costal arch, respectively. Resected part of the diaphragm (b)
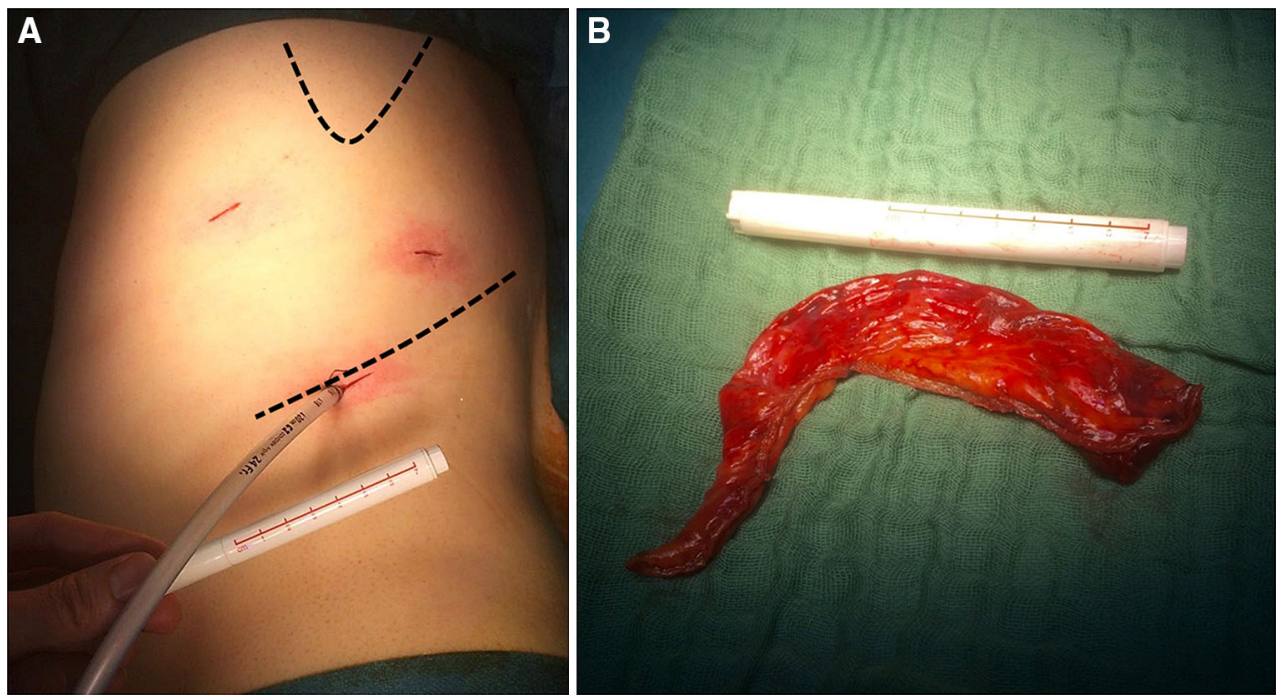
Fig. 2 Preoperative chest X-ray showing an elevated right hemidiaphragm (a). Chest X-ray 3 days after thoracoscopic plication showing a flattened right hemidiaphragm with complete lung re-expansion on the right side (b)
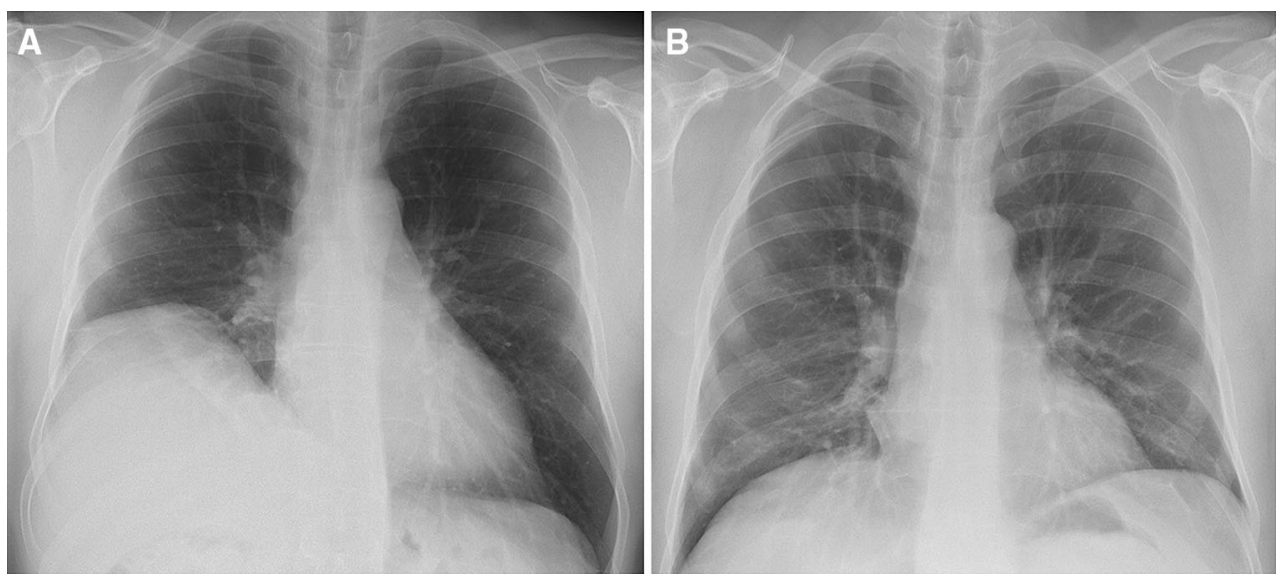

corresponding lung was achieved in all patients (Fig. 2). In one patient, an 86-year-old female with concomitant transdiaphragmatic intercostal hernia, the operation time was $120 \mathrm{~min}$ since she had to undergo additional hernia mobilization and repair during the same procedure. In the same patient, noninvasive ventilatory support was necessary on POD 5 for $24 \mathrm{~h}$ due to lower lobe atelectasis on the contralateral side with consecutive respiratory insufficiency. The remaining patients showed an uneventful postoperative course. Patients were discharged after a mean duration of 3.2 days (range $2-5, \mathrm{SD} \pm 0.97$ ), except for the older female patient with transdiaphragmatic hernia, who stayed for 14 days. All patients were relieved from their symptoms, and the abovementioned lady, who required oxygen treatment before the procedure, was without oxygen within one month after surgery. No patient showed recurrence or even breakage of the suture line after a mean follow-up of 7.5 months (range 4-12, $\mathrm{SD} \pm 3.28$ ) after surgery. All patients underwent a routine follow-up visit including lung function testing 3 months after the procedure. At the time of follow-up, all patients did well without any pain medication treatment and showed significant improvements in lung function values. FEV1 showed a mean increase of $540 \mathrm{ml}$ (range $220-740 \mathrm{ml}$, $\mathrm{SD} \pm 193 \mathrm{ml}$ ), and the same was true for FVC with a mean increase of $776 \mathrm{ml}$ (range $680-980 \mathrm{ml}$, $\mathrm{SD} \pm 121 \mathrm{ml}$ ), respectively. Lung function changes did not differ significantly between right- and left-sided plications.

\section{Discussion}

The introduction of thoracoscopy for diaphragmatic plication was first described in 1995 by Gharagozloo et al. [2], and many adaptions to this technique have been made in the years that followed. Nowadays most surgeons prefer a video-assisted thoracoscopic approach involving a minithoracotomy. If the operation is performed in a completely thoracoscopic way, full-thickness figure of eight sutures [5] seem to be most appropriate and applicable. Furthermore the application of an Endostitch device with a pledgeted suturing technique has been recently described in order to facilitate the whole procedure [6]. Despite all the efforts that have been made to simplify the minimally invasive approach, none of the abovementioned techniques has been widely accepted so far.

Suturing seems to be the main problematic part of the procedure, since it is time-consuming and more complicated in thoracoscopic than in open surgery. Furthermore concerning the safety issue, injury to the intraabdominal organs is the main concern when applying full-thickness sutures to the diaphragm.

Recently, Kara et al. [7] described their technique of stapling of the diaphragm by means of thoracoscopy, involving a $2-3-\mathrm{cm}$ utility incision.

With our technique, a completely thoracoscopic approach was easily possible in all patients. In addition we only used $5 \mathrm{~mm}$ ports in the intercostal spaces, and the largest port, (12 mm) which was required for stapling, was introduced below the costal arch. The advantages are less pain and an optimal position for stapling of the diaphragm, since the port is exactly on the same level and in line with the diaphragm. Since we use a completely portal approach without utility incision, the intraabdominal organs are pushed down with the help of $\mathrm{CO} 2$ insufflation and an anti-Trendelenburg position of the patient, which makes the intraabdominal organs much less susceptible to accidental lesions during surgery. Furthermore by rolling up the diaphragm on a grasper before stapling and additionally gently pushing away the intraabdominal organs with the stapler before completely closing and firing it, the risk for any visceral injury is absolutely minimized. Since breakage of the staple line is a major concern when using a stapling device for this procedure, we strongly 
advise to reinforce the staple line with a suture. This brings us to another main advantage of our technique: Suturing is quite easy after the diaphragm has been stapled, since the desired tension and flattening of the diaphragm is already achieved and the abundant diaphragm slack is already removed. Thus, a running suture, requiring only two knots or even a barbed suture without the need for any knot-tying (video 2), can be used. Whether additional suturing of the staple line is necessary remains a debatable issue, especially when a stapling device is used that leaves three lines of staples in place after resection. But since breakage of diaphragmatic staple lines has been described in the literature [8] and since it would result in severe consequences for the patient, we believe that an additional reinforcement with a suture should be performed in all cases.

Although we do not have a longer-term follow-up on our patients yet, we believe that our proposed technique is easily applicable and allows a less invasive, safe and effective way to perform diaphragmatic plication. Certainly larger studies are needed to prove the reproducibility of our technique and to confirm our results.

\section{Compliance with ethical standards}

Conflict of interest All authors declare that they have no conflict of interest.

\section{References}

1. Qureshi A (2009) Diaphragm paralysis. Semin Respir Crit Care Med 30(3):315-320

2. Gharagozloo F, McReynolds SD, Snyder L (1995) Thoracoscopic plication of the diaphragm. Surg Endosc 9:1204-1206

3. Mouroux J, Padovani B, Poirier NC et al (1996) Technique for the repair of diaphragmatic eventration. Ann Thorac Surg 62:905-907

4. Moon SW, Wang YP, Kim YW et al (2000) Thoracoscopic plication of diaphragmatic eventration using endostaplers. Ann Thorac Surg 70(1):299-300

5. Kim Do H, Joo Hwang J, Kim KD (2007) Thoracoscopic diaphragmatic plication using three $5 \mathrm{~mm}$ ports. Interact CardioVasc Thorac Surg 6(3):280-281

6. Dunning J (2015) Thoracoscopic diaphragm plication. Interact CardioVasc Thorac Surg 20(5):689-690

7. Kara HV, Roach MJ, Balderson SS, D'Amico TA (2015) Thoracoscopic diaphragm plication. Ann Cardiothorac Surg 4(6):573-575

8. Minegishi K, Nakano T, Shibano T et al (2014) Diaphragmatic hernia complicated with diaphragmatic resection by automatic stapling device. Kyobu Geka 67(11):959-962 